\title{
Microwave Active Filters Based on Transversal and Recursive Principles
}

\author{
CHRISTEN RAUSCHER, SENIOR MEMBER, IEEE
}

\begin{abstract}
Transveral and recursive filter concepts, normally associated with discrete-time applications, are adapted for implementation in the microwave frequency range. Principal focus is on microwave active filter designs that permit realization of wide bandwidths, with microwave transistors used as active elements. A transversal-type and a recursive-type experimental filter, both of which exhibit 9-15-GHz bandpass responses, serve to illustrate aspects of physical implementation, while offering a convenient basis for comparing the two different approaches.
\end{abstract}

\section{INTRODUCTION}

$\mathrm{T}$ HE MENTION OF active filters invariably brings to mind the familiar concepts employed at very low frequencies, involving operational amplifiers embedded in lumped-element feedback and coupling circuitry. Among the desirable attributes of these filters are their ability to compensate for losses associated with passive circuit elements and their ability to provide compact physical realizations of higher order transfer functions. Achieving these attributes can be equally relevant in the high-frequency range. A case of particular interest relates to filter requirements affiliated with GaAs monolithic circuit implementation of microwave and millimeter-wave subsystems. Unfortunately, the direct transposition of low-frequency design principles to the microwave range is impeded by the lack of appropriate broad-band, high-gain devices to perform operational amplifier functions. Gain itself is a major concern, imposing constraints on possible design options, but it is really time delay intrinsic to microwave active devices which tends to be the primary limiting factor. Certain operational-amplifier-based concepts could be adapted for implementation in the lower microwave frequency range, yet have received little attention to date. Nevertheless, judging by the literature, there has been sporadic interest in alternative microwave active filter concepts. Reported work [1]-[3] has concentrated on replacing individual circuit elements in passive filter structures with microwave active substitutes. These substitutes typically make use of transistors and are designed to exhibit, over a limited frequency range, behaviors similar to those of the reactances and resonators they replace, but provide higher $Q$ performance than their passive counterparts. They appear to be mainly suited for relatively narrow-band situations.

Manuscript received March 18, 1985; revised June 10, 1985

The author is with the Electronics Technology Division of the Naval Research Laboratory, Code 6851, Washington, DC 20375-5000. He is currently on sabbatical leave at the Los Alamos National Laboratory, Los Alamos, NM 87545.
In contrast to the lumped-element and quasi-lumped-element filters mentioned above, the present study focuses on distributed active filter concepts. Although these concepts are normally associated with applications in the digital domain, they can also be adapted to address analog filter needs. They are of particular interest in the present context because of their potential for more easily coping with active device constraints relative to gain and time delay at microwave frequencies. The distributed filter structures to be considered are divided into two basic categories. The first category comprises circuits of the transversal type which, aside from parasitic feedback effects, employ feedforward techniques exclusively. A flow graph representation of such a filter is shown in Fig. 1a. The underlying design concept displays certain similarities with familiar microwave distributed amplifiers. The fundamental distinction between the two kinds of circuits is that, in the amplifier case, individual signal components are all combined in-phase, whereas, in the filter case, the frequencyselective overall response is derived by combining signal components with different amplitudes and frequencydependent phase delays. The second category encompasses filters of the recursive type. As the name indicates, these filters are based on the use of feedback, generally involving transversal principles simultaneously. Filter responses are again derived through interaction between signal components with appropriate amplitude and phase relationships. The flow graph example in Fig. 1(b) depicts one of the numerous ways of representing such a filter. The additional design freedom offered by the presence of feedback generally translates into more compact circuit realizations. Although the recursive structures give the appearance of being potentially more susceptible to instability than transversal-type circuits, parasitic feedback within typical microwave active two-port devices tends to disallow a meaningful distinction in practical design situations.

The study outlined in the following aims at exploring the use of both transversal and recursive principles in deriving practical filter designs for microwave applications. Emphasis, thereby, is placed on achieving wide bandwidths of up to an octave, in conjunction with sharp cutoff characteristics at the band edges. Compact filters of this kind are required, for instance, in monolithic full-band millimeterwave receivers to process the ultra-wide-band microwave IF signals that result. The general approach pursued here is to seek realistic and economic utilization of distributed filter concepts, while keeping the number of required active 


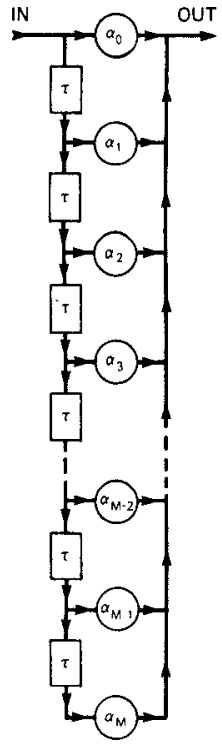

(a)

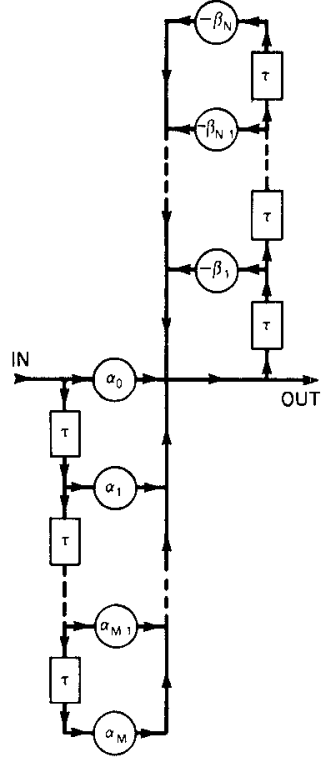

(b)
Fig. 1. Flow graph examples of distributed filters: (a) transversal filter and (b) recursive filter.

devices within reasonable bounds. One of the options considered to achieve this goal is to include, where appropriate, conventional passive filter concepts in the overall distributed active designs. Two experimental filter examples, one in each category, will serve to illustrate this hybrid approach.

\section{Microwave Filiters of the Transversal Type}

\section{A. General Design Approach}

Transversal filters in their classical form are direct implementations of Fig. 1(a), involving tapped delay lines with constant delay increments, as well as means for amplitude-weighting and combining of the various delayed signal components. This type of filter is most commonly affiliated with discrete-time applications that specifically require maintaining constant group delay across the frequency band of interest. To obtain the necessary linear phase characteristics, the amplitude distribution of the weighted signal components must exhibit symmetry relative to the center of the structure. Symmetry can then be utilized to economize in the number of weighting elements needed by folding the structure around its center. In the present context, however, constant group delay will not be considered a relevant issue, thereby providing the freedom to resort to asymmetric filter designs where advantageous. Design freedom could be further enhanced by waiving the need for equal time delay increments as carried over from analogous discrete-time implementations. From the point of view of developing practical design procedures, however, the constant delay increment assumption remains desirable. Subsequent derivations will hence be based on delay increments which are either all equal or are otherwise multiples of a common minimum delay length. The complex transfer function of a transversal filter with $M+1$ weighting elements, in terms of the angular frequency $\omega$, is thus

$$
H_{T}(j \omega)=\sum_{m=0}^{M} \alpha_{m} \cdot e^{-\jmath 2 \pi m \omega / \omega_{s}}
$$

with the frequency $\omega_{s}$, at which the periodic transfer function repeats itself, defined as

$$
\omega_{s}=2 \pi / \tau \text {. }
$$

The coefficients $\alpha_{m}$ represent the amplitude weighting factors, and $\tau$ refers to the constant time delay interval introduced in Fig. 1(a).

In a practical microwave implementation, the overall filter network will invariably encompass circuit components that are bandlimited in one way or another. Furthermore, as alluded to in the Introduction, it may be desirable to intentionally include conventional filter segments in an otherwise strictly transversal design. If such auxiliary filtering effects can be factored out and represented by the supplementary transfer function $H_{S}(j \omega)$, then the composite filter response may be written as

$$
H(j \omega)=H_{S}(j \omega) \cdot H_{T}(j \omega) .
$$

The decomposition implies that all transversal signal components are equally exposed to the auxiliary effects. This tends to be generally the case in the kinds of circuits most suitable for microwave implementation, whence assumption (3) is adopted throughout the derivations to follow.

The basic design task is thus to determine the combination of $\omega_{s}$ and parameters $\alpha_{m}, m=0,1, \cdots, M$, which permits the composite function $H(j \omega)$ to best approximate a prescribed target response function $G(j \omega)$. For the purposes of the present discussions, $G(j \omega)$ shall be assumed to represent a bandpass filter for which only the magnitude response matters, with the underlying approach applicable to most any other transversal-type filter as well. Viewing (1) as a truncated Fourier expansion then leads to

$$
\begin{array}{r}
\alpha_{m}=\frac{1}{\omega_{s}} \cdot \int_{\omega_{A}}^{\omega_{B}} \frac{|G(j \omega)|}{\left|H_{S}(j \omega)\right|} \cdot e^{+j 2 \pi(m-M / 2) \omega / \omega_{s}} \cdot d \omega, \\
m=0,1, \cdots, M
\end{array}
$$

whereby $\omega_{A}$ and $\omega_{B}$ correspond to the lower and upper bandpass cutoff frequencies, respectively, beyond which $|G(j \omega)|$ is set equal to zero. In this context, both $\omega_{s}$ and the magnitude function $\left|H_{S}(j \omega)\right|$ are considered to be predetermined by other circumstances. An alternative expression is obtained by defining $M+1$ uniformly spaced frequency points within the interval $0 \leqslant \omega<\omega_{s}$, and then substituting summation for integration in (4)

$$
\begin{gathered}
\alpha_{m}=\frac{1}{M+1} \cdot \sum_{k=K_{A}}^{K_{B}} \frac{\left|G\left(j \frac{k}{M+1} \omega_{s}\right)\right|}{\left|H_{S}\left(j \frac{k}{M+1} \omega_{s}\right)\right|} \\
\cdot e^{+\jmath 2 \pi k(m-M / 2) /(M+1)}, \quad m=0,1, \cdots, M .
\end{gathered}
$$

The index limits $K_{A}$ and $K_{B}$ bracket the passband values 
of $k$. The values of the coefficients $\alpha_{m}$ represent complex numbers, in general. But for even periodic transfer functions, like the ones to be focused on here, all the coefficients become real. Of particular interest for implementing wide bandpass filters are even responses of the distributed low-pass type and the distributed high-pass type. These complementary responses exhibit double symmetry, with passbands and stopbands centered around even multiples and odd multiples of $\omega_{s} / 2$, respectively. Symmetry also expresses itself in terms of the coefficients, with

$$
\alpha_{m}=\alpha_{M-m}, \quad \text { for all } m .
$$

\section{B. Simplifying Approximations}

Realization of a given target response invariably leads to coefficients with both positive and negative signs. This can be accomplished in a microwave environment by splitting the incident signal into two signals of opposite phase and then utilizing separate delay lines from which to derive transversal signal components of required time delay and sign [4]. An alternative would be to employ only a single feed line, but satisfy the sign requirements by engaging microwave active devices in both inverting and noninverting configurations. From a practical point of view, neither of these solutions appears very attractive: The former approach tends to consume a disproportionate amount of real estate, while the latter solution sacrifices design uniformity by typically having to accept amplifier sections with different reflection, isolation, and delay characteristics. Options were thus explored that would permit evasion of the underlying dual-sign problem. One such option, which particularly pertains to filters with passbands centered around $\omega_{s} / 2$, is to eliminate all opposite-sign contributions to the sum expression (1) by substituting approximate terms for them according to

$$
\begin{aligned}
& \alpha_{m} \cdot e^{-j 2 \pi m \omega / \omega_{s}} \\
& \approx-\alpha_{m} \cdot \gamma \cdot\left\{e^{-\jmath 2 \pi(m-1) \omega / \omega_{s}}+e^{-\jmath 2 \pi(m+1) \omega / \omega_{s}}\right\}, \\
& \quad \text { for } \alpha_{m} / \alpha_{M / 2}<0 \text { and } m>0 .
\end{aligned}
$$

Without loss of generality, it is thereby tacitly assumed that $M$ is an even number. Systematic substitution leads to a new set of $M+1$ one-sign-only coefficients

$$
\begin{aligned}
& \bar{\alpha}_{0}=\bar{\alpha}_{M}=\alpha_{M / 2} \cdot\left\{0.5 \cdot\left(\left|\frac{\alpha_{0}}{\alpha_{M / 2}}\right|+\frac{\alpha_{0}}{\alpha_{M / 2}}\right)\right. \\
& \left.+\frac{\gamma}{2} \cdot\left(\left|\frac{\alpha_{1}}{\alpha_{M / 2}}\right|-\frac{\alpha_{1}}{\alpha_{M / 2}}\right)\right\} \\
& \bar{\alpha}_{1}=\bar{\alpha}_{M-1}=\alpha_{M / 2} \cdot\left\{0.5 \cdot\left(\left|\frac{\alpha_{1}}{\alpha_{M / 2}}\right|+\frac{\alpha_{1}}{\alpha_{M / 2}}\right)\right. \\
& +0.5 \cdot\left(\left|\frac{\alpha_{0}}{\alpha_{M / 2}}\right|-\frac{\alpha_{0}}{\alpha_{M / 2}}\right) \\
& \left.+\frac{\gamma}{2} \cdot\left(\left|\frac{\alpha_{2}}{\alpha_{M / 2}}\right|-\frac{\alpha_{2}}{\alpha_{M / 2}}\right)\right\}
\end{aligned}
$$

$$
\begin{gathered}
\bar{\alpha}_{m}=\bar{\alpha}_{M-m}=\alpha_{M / 2} \cdot\left\{0.5 \cdot\left(\left|\frac{\alpha_{m}}{\alpha_{M / 2}}\right|+\frac{\alpha_{m}}{\alpha_{M / 2}}\right)\right. \\
+\frac{\gamma}{2} \cdot\left(\left|\frac{\alpha_{m-1}}{\alpha_{M / 2}}\right|-\frac{\alpha_{m-1}}{\alpha_{M / 2}}\right) \\
\left.+\frac{\gamma}{2} \cdot\left(\left|\frac{\alpha_{m+1}}{\alpha_{M / 2}}\right|-\frac{\alpha_{m+1}}{\alpha_{M / 2}}\right)\right\}, \\
m=2,3, \cdots, M / 2-1 \\
\bar{\alpha}_{M / 2}=\alpha_{M / 2} .
\end{gathered}
$$

An empirical value for $\gamma$ in the vicinity of 0.6 renders approximation (7) and the above substitution coefficients useful for bandwidths up to about an octave. Furthermore, if these coefficients are small relative to the main transmission term $\alpha_{M / 2}$ and if a break with the linear phase tradition is permissible, then a respective pair of coefficients $\left(\alpha_{m}, \alpha_{M-m}\right)$ may be replaced by a single-sided approximation wherein one of the coefficients is deleted and the other assumes a double role. And, if the contribution by a term is judged negligible, it may be convenient to delete it altogether. Systematic exercise of these approximations leads to a one-sided sparse transversal array.

The above substitutions and deletions of course result in some passband deviations from the original response, as design parameters yield to constraints. Computer optimization can then be employed to adjust the pertinent parameters and bring the filter response back in line with specifications. This is accomplished at the same time as ripple introduced through truncation in the Fourier expansion (1) is smoothed out and microwave circuit parasitic effects are compensated for. A repetition of the procedure with a higher value of $M$ might be indicated if it turns out that there are too few design variables to cope with deviations.

The equal-sign approximations (8) break down at remote stopband frequencies. At points where zeros of transmission should occur, the modified transversal components actually combine to produce maximum transmission. A window function must thus be introduced to help control out-of-band behavior. It is thereby crucial to satisfy the added requirement without compromising the benefits of reduced circuit complexity derived from the approximations. This may be conveniently accomplished through assignment of windowing responsibilities to the individual microwave active devices within the overall transversal structure and by tailoring the associated input and output matching circuitries accordingly. And finally, depending on the application, the overall circuit may be augmented by conventional filter sections to assist in subduing out-ofband transmission peaks, in shaping filter skirts, and in supplying designated zeros of transmission.

\section{Physical Implementation}

When it comes to physically realizing a transversal-type filter there are of course many ways of achieving this. Fig. 2 displays a generalized block diagram of a microwave 


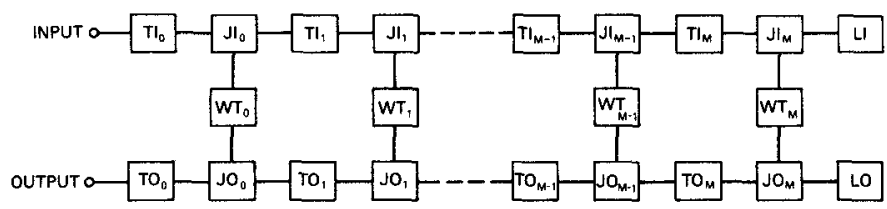

Fig. 2. Block diagram of a microwave transversal filter.

single-feed structure, composed of input and output transmission elements $T I_{m}$ and $T O_{m}$, input and output junction elements $J I_{m}$ and $J O_{m}$; weighting elements $W T_{m}$, and optional line terminations $L I$ and $L O$. The principal purpose of the transmission elements is to provide the required time delays. In their simplest form, they consist merely of uniform transmission-line segments, but may include, where appropriate, conventional passive filter segments and active devices to help conform with window function requirements and to aid in realizing the desired phase and amplitude relationships among the various transversal signal components. Likewise, the junction elements may consist of only simple lossless three-way junctions, or they may comprise more involved splitters and combiners that utilize resistors to improve isolation and matching conditions. If the junction elements do not provide sufficient isolation on their own between output and input, the weighting elements will need to be directional if the filtering process is to be genuinely transversal. The use of transistors in either inverting or noninverting configurations represents an obvious choice, with amplitude weighting conveniently accomplished through bias control and impedance matching.

In the course of this study, a variety of different combinations of the above-outlined options were explored in $X$ and $K u$-band designs. Submicron gate-length GaAs FET's were relied on as active elements throughout the investigation. Emphasis was on filter solutions that make effective use of a minimum number of active devices, that efficiently process available incident signal power, and that readily provide compact and realizable circuits. One of the main difficulties encountered in practical designs is related to parasitic interactions between individual transistors and the rest of the circuit. These interactions result from residual reflections off each impendance-matched transistor input and output, and from device-intrinsic feedback. A lower bound on parasitic effects is predominantly determined by bandwidth requirements and by practical restrictions on the amount of circuit area and the amount of composite time delay that may be afforded for each active device. The characteristics of a number of filter examples were simulated, based on the use of octave-bandwidth windowing amplifiers. Each of these amplifiers consisted of either a single half-micron or a single quartermicron transistor which was impedance-matched to achieve minimum return losses at input and output between 8 and $10 \mathrm{~dB}$, depending on design. Although these reflections by themselves would be considered harmless, several of them interacting with each other in a circuit containing numerous active devices and numerous three-way junctions can result in substantial overall gain variations and also lead to stability problems when combined with parasitic feedback through the transistors. The problem is amplified by dealing with structures that are inherently of considerable electrical length, causing rapid relative phase shifts between parasitic signal components that are difficult to handle over a wide band. An obvious solution is to prevent parasitic signals from interacting with the principal transversal process by designing the isolation properties of each junction element accordingly. Depending on how critical losses through a particular junction in the main signal direction are for maintaining overall filter performance, the junction element might involve a Wilkinson divider or combiner, or merely require the use of a resistive series tap element to provide adequate isolation. If sufficient isolation is provided in this manner, there is actually no need anymore to employ directional weighting elements, freeing up transistors for strategically more important tasks within an overall filter design.

Design considerations are obviously dominated by transistor-related constraints, as might be expected in any situation encompassing microwave active semiconductor devices. Of concern are parasitic effects, preservation of valuable gain, efficient utilization of substrate area, reliability, and cost. Of particular concern is the task of properly accounting for the rather complex two-port characteristics of each transistor by itself. Accommodation of these concerns requires the targeted exploitation of available design options comprising, in the present context, the appropriate use of windowing amplifiers and passive filter segments in conjunction with one-sign-only and sparse approximations. Due to the diversity of issues involved, the process of determining the best mix of available options for given types of transistors must invariably depend on empirical elements to some extent. This tends to stand in the way of deriving generalized design algorithms and elegant closed-form solutions as are common in familiar passive filter approaches. The inconvenience of having to rely on empirical procedures with extensive use of computer optimization is rewarded, however, in terms of enhanced performance potential. The following experimental example should help illustrate the underlying basic design philosophy.

\section{Experimental Example}

The objective adopted for the experiment was to obtain a triple-hump bandpass response between cutoff frequencies 9 and $15 \mathrm{GHz}$, while minimizing transmission at designated stopband frequencies within the $5-8-\mathrm{GHz}$ and $16-19-\mathrm{GHz}$ intervals. After weighing available options, it was decided to implement the filter as a cascade connection of a strictly transversal filter section, a conventional filter portion, and a gain section. Based on the above comments on isolation, it was furthermore concluded that, in the present application, it would not constitute an effective utilization of transistors to employ them as directional weighting elements.

A general-purpose broad-band gain section was first developed. The section comprises an Avantek M126 GaAs 
FET matched at input and output to achieve a flat gain of approximately 8 to $9 \mathrm{~dB}$ between 8 and $16 \mathrm{GHz}$, with distinct gain roll-off beyond those points to be utilized for windowing purposes. From a sensitivity point of view, it was judged essential to have the bandwidth of the gain section exceed the intended filter bandwidth so as to minimize tolerance effects on critical filter skirts due to variations in transistor characteristics. For the conventional filter portion, a simple auxiliary $50-\Omega$ filter segment was selected, consisting merely of two parallel $100-\Omega$ transmission lines with lengths of a quarter wave and of five quarter waves at band center $(12 \mathrm{GHz})$, respectively. The purpose of this filter is to aid the transversal process in shaping the filter characteristics by introducing zeros of transmission at $6,8,16$, and $18 \mathrm{GHz}$.

As for the design of the transversal section, the previously outlined procedure was followed. The supplementary transfer function $H_{S}(j \omega)$ introduced in factorization (3) corresponds, in the present example, to the composite transfer function of the cascade of the auxiliary passive filter and the gain section-a known function at this point in the design process. After calculating a representative set of $\alpha$-coefficients according to relationship (5), the design was then systematically whittled down to the bare minimum by applying the one-sign-only and single-sided approximations, and then deleting all but the components most relevant to forming filter skirts and defining passband behavior. The transversal section with bandpass-type characteristics centered around $\omega_{s} / 4 \pi=12 \mathrm{GHz}$ thus emerged as a three-branch structure. The electrical lengths at band center for each branch, referenced to the signal path exhibiting the shortest delay, were $0,(5 / 2) \pi$, and $4 \pi$, respectively. Impedance transforming transmission-line sections, asymmetrical single-section Wilkinson splitters and combiners, and a series tap resistor were used to achieve the necessary amplitude distribution among the three transversal signal components, as well as output-toinput isolation. The composite design was then subjected to a round of numerical circuit optimization to even out imperfections that resulted from the approximation and deletion processes.

The microstrip hardware realization of the three segment filter is depicted in Fig. 3. The circuit is implemented on a 0.25 -mm-thick fiberglass-reinforced Teflon substrate, with coaxial $50-\Omega$ input and output connectors. For ease of reference, a schematic of this circuit is provided in Fig. 4, together with a list of the various circuit element values. The transversal filter section encompasses transmission-line segments $T L_{1}$ through $T L_{17}$, as well as series tap resistor $R_{1}$ and Wilkinson isolation resistors $R_{2}$ through $R_{4}$. The passive filter section is represented by $T L_{18}$ and $T L_{19}$, with the remaining circuit elements associated with the gain section. The measured filter response, obtained with the help of a few minor trimmings to compensate for statistical chip-to-chip variations in transistor characteristics, is compared in Fig. 5 with the calculations. The only transistor used in this circuit, namely the Avantek M126 device employed in the gain section, was biased at half $I_{D S S}$ and

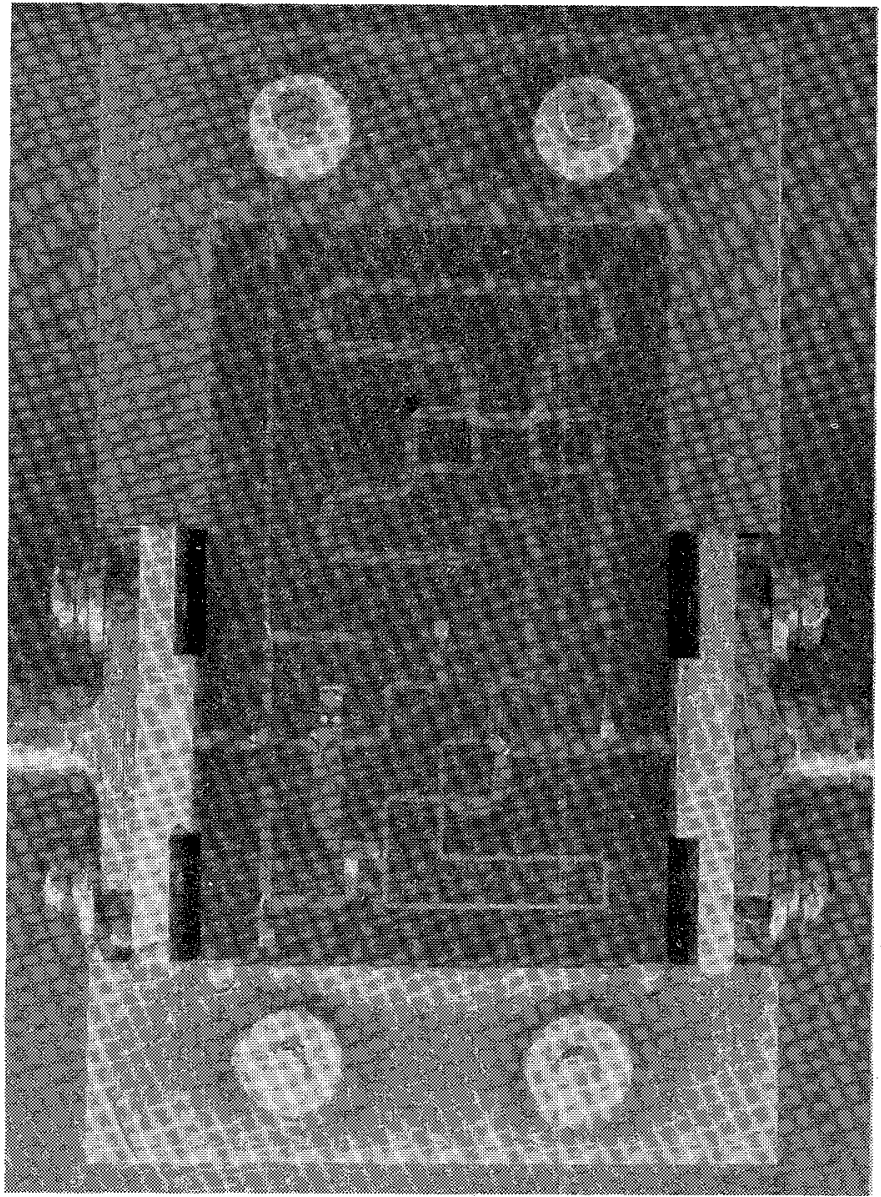

Fig. 3. Experimental 9-15-GHz transversal-type bandpass filter, implemented on a 0.25 -mm-thick fiberglass-reinforced Teflon substrate. The $50-\Omega$ input port is on the right and the $50-\Omega$ output port on the left.

at a drain-source voltage $V_{D S}=+3.0 \mathrm{~V}$. Also indicated in Fig. 5 is the frequency response of the gain section by itself.

With focus on the development of basic filter concepts, issues relating to noise and nonlinear performance were not given specific consideration. In a general situation, these issues invariably require that bias levels as well as input and output impedance matching conditions be optimized for individual transistors in a given circuit configuration to arrive at a satisfactory compromise between competing objectives. Transversal filters that contain active branch elements tend to permit relatively straightforward treatment due to absence of major feedback. As active device participation in the present example ended up being limited to a post-amplification function, nonlinear behavior of the composite filter should closely resemble that of a typical GaAs FET amplifier stage, whose device is biased at half- $I_{D S S}$ and matched to a $50-\Omega$ load at the output. Analysis and measurement of such behavior-the subject of earlier investigations [5], [6] - was hence not pursued in detail. With respect to noise performance, the overall noise figure of the filter was spot-checked across the $9-15-\mathrm{GHz}$ passband at one- $\mathrm{GHz}$ intervals. The measured results describe a U-shaped response with maximum readings of 11.6 


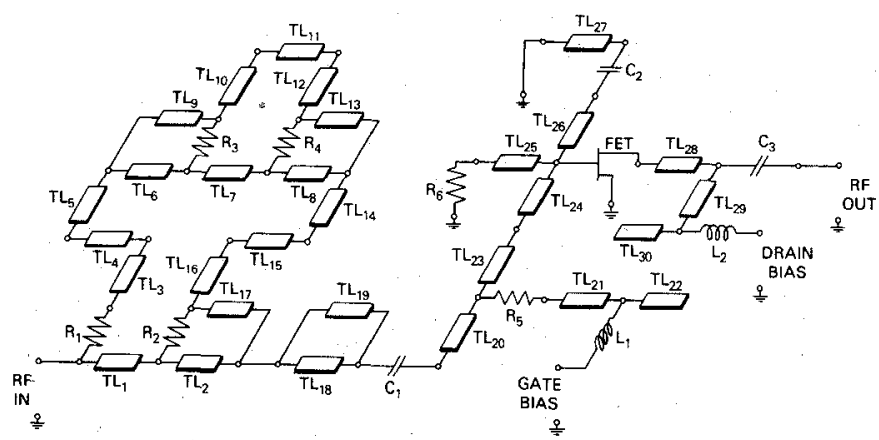

Fig. 4. Schematic circuit diagram of the experimental transversal-type bandpass filter. The values of characteristic impedance $Z_{0}$ and electrical length $\theta$ at $12 \mathrm{GHz}$ for the different uniform transmission-line sections are

\begin{tabular}{|c|c|c|c|c|c|}
\hline$T L_{1}:$ & $Z_{0}=42 \Omega$, & $\theta=90^{\circ}$ & $T L_{16}:$ & $Z_{0}=70 \Omega$ & $\theta=90^{\circ}$ \\
\hline$T L_{2}:$ & $Z_{0}=52 \Omega$, & $\theta=90^{\circ}$ & $T L_{17}:$ & $Z_{0}=105 \Omega$, & $\theta=90^{\circ}$ \\
\hline$T L_{3}:$ & $Z_{0}=105 \Omega$, & $\theta=90^{\circ}$ & $T L_{18}$ : & $Z_{0}=100 \Omega$, & $\theta=90^{\circ}$ \\
\hline$T L_{4}:$ & $Z_{0}=45 \Omega$, & $\theta=90^{\circ}$ & $T L_{19}:$ & $Z_{0}=100 \Omega$ & $\theta=450^{\circ}$ \\
\hline$T L_{5}:$ & $Z_{0}=50 \Omega$, & $\theta=45^{\circ}$ & $T L_{20}:$ & $Z_{0}=50 \Omega$ & $\theta=30^{\circ}$ \\
\hline$T L_{6}:$ & $Z_{0}=78 \Omega$, & $\theta=90^{\circ}$ & $T L_{21}:$ & $Z_{0}=65 \Omega$ & $\theta=100^{\circ}$ \\
\hline$T L_{7}:$ & $Z_{0}=5$ & $\theta=45^{\circ}$ & $T L_{22}$ & $Z_{0}=65 \Omega$ & $\theta=90^{\circ}$ \\
\hline$T L_{8}:$ & $Z_{0}=78 \Omega$, & $\theta=90^{\circ}$ & $T L_{23}$ : & $Z_{0}=50 \Omega$ & $\theta=30^{\circ}$ \\
\hline$T L_{9}:$ & $Z_{0}=65 \Omega$, & $\theta=90^{\circ}$ & $T L_{24}$ : & $Z_{0}=35 \Omega$, & $\theta=78^{\circ}$ \\
\hline$T L_{10}$ : & $Z_{0}=5$ & $\theta=90^{\circ}$ & $T L_{25}:$ & $Z_{0}=90 \Omega$ & $\theta=135^{\circ}$ \\
\hline$T L_{11}:$ & $Z_{0}=55 \Omega$ & $\theta=405^{\circ}$ & $T L_{26}$ : & $Z_{0}=40 \Omega$, & $\theta=12^{\circ}$ \\
\hline$T L_{12}:$ & $Z_{0}=50 \Omega$, & $\theta=90^{\circ}$ & $T L_{27}:$ & $Z_{0}=40 \Omega$ & $\theta=32^{\circ}$ \\
\hline$T L_{13}$ : & $Z_{0}=65 \Omega$, & $\theta=90^{\circ}$ & $T L_{28}$ & $Z_{0}=95 \Omega$ & $\theta=34^{\circ}$ \\
\hline$T L_{14}:$ & $Z_{0}=60 \mathrm{~s}$ & $\theta=90^{\circ}$ & $T L_{29}$ & $Z_{0}=75 \Omega$ & $\theta=89^{\circ}$ \\
\hline$T L_{15}:$ & $Z_{0}=70 \Omega$ & $\theta=360^{\circ}$ & $T L_{30}:$ & $Z_{0}=75 \Omega$, & $\theta=90^{\circ}$ \\
\hline
\end{tabular}

The values of the lumped elements are

$$
\begin{array}{ll}
R_{1}=250 \Omega & C_{1}=C_{3}=10 \mathrm{pF} \\
R_{2}=R_{3}=R_{4}=R_{6}=100 \Omega & C_{2}=20 \mathrm{pF} \\
R_{5}=400 \Omega & L_{1}=L_{2}=12 \mathrm{nH}
\end{array}
$$

The transistor is a sub-half-micron Avantek M126 device.

$\mathrm{dB}$ and $11.5 \mathrm{~dB}$ at the lower and upper passband edges, respectively, and a minimum figure of $7.2 \mathrm{~dB}$ at $13 \mathrm{GHz}$. The numbers reflect the fact that the filter was optimized for gain performance only, with little concern for the effects of resistive matching at the transistor input or for the implications of favoring post-amplification over preamplification for topological convenience. Nevertheless, the measurements do possess some comparative value relative to the recursive example discussed later.

\section{MicrowaVe Filters of the Recursive Type}

\section{A. General Approach}

As indicated in Fig. 1(b), a recursive filter may be regarded as a tandem combination of a transversal section and a feedback-only section. The presence of feedback constitutes a very powerful extension relative to a strictly transversal process, permitting poles of transmission of arbitrarily high $Q$-factors to be realized. Introduction of such poles invariably impacts on sensitivity and stability considerations so that an overall design will normally involve some competition among performance criteria. Filter characteristics are again the result of interaction

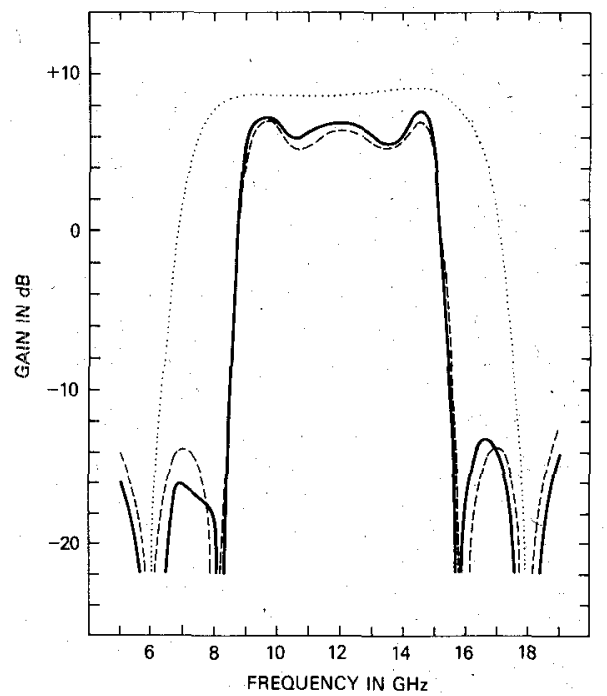

Fig. 5. Frequency characteristics of the transversal-type experimental filter: - measured filter response, -..-- calculated filter response, ... calculated characteristics of the amplifier section by itself.

between time-delayed signal components. Under the assumption that the particular time delays are multiples of some common minimum delay $\tau=2 \pi / \omega_{s}$, the recursive transfer function of a structure with $M+1$ feedforward branches and $N$ feedback branches can be written in the form

$$
H_{R}(j \omega)=\frac{\sum_{m=0}^{M} \alpha_{m} \cdot e^{-j 2 \pi m \omega / \omega_{s}}}{1+\sum_{n=1}^{N} \beta_{n} \cdot e^{-j 2 \pi n \omega / \omega_{s}}}
$$

As in the transversal case, the basic recursive process will be allowed assistance from supplementary filter sections represented by a corresponding transfer function $H_{S}(j \omega)$. The composite filter response $H(j \omega)$ is thereby assumed to be of the factorial form

$$
H(j \omega)=H_{S}(j \omega) \cdot H_{R}(j \omega) .
$$

With $H_{S}(j \omega)$ normally predetermined by constraining circumstances or derived so as to conform with windowing requirements, the design task is to find coefficient values $\alpha_{m}, m=0,1, \cdots, M$, and $\beta_{n}, n=1,2, \cdots, N$, that yield the best approximation by $H(j \omega)$ to a prescribed target response function $G(j \omega)$. There are several methods for accomplishing this task. Two such methods, based on techniques commonly used in discrete-time applications [7], shall be briefly outlined in the following.

1) Impulse Response Method: This approach starts out with the use of conventional, well-established s-plane synthesis techniques to derive a rational function approximation $\bar{H}_{R}(s)$ that will represent the magnitude of the target response $G(j \omega) / H_{S}(j \omega)$ of the recursive filter section along the $j \omega$-axis. Expressed in terms of partial fractions, this leads to

$$
\bar{H}_{R}(s)=\sum_{i=1}^{N_{P}} \frac{r_{i}}{s-s_{i}}
$$


whereby $r_{i}$ and $s_{2}$ denote the residue and the location of the $i$ th $s$-plane pole, respectively. An alternative expression for $\bar{H}_{R}(s)$ is

$$
\bar{H}_{R}(s)=\sum_{i=1}^{N_{P}} r_{\imath} \int_{0}^{\infty} e^{\left(s_{t}-s\right) t} \cdot d t
$$

The integral is subsequently approximated by a finite-element summation that relies on dividing the time axis into equal-length intervals of $2 \pi / \omega_{s}$ duration. ( $\omega_{s}$ is assumed to be predetermined at this point in the design.) The result is a rational function of exponential terms that conforms to definition (9):

$$
H_{R}(s)=\frac{2 \pi}{\omega_{s}} \cdot \sum_{i=1}^{N_{P}} \frac{r_{t}}{1-e^{\left(s_{i}-s\right) 2 \pi / \omega_{s}}} .
$$

Substitution of $j \omega$ for $s$ and rearrangement of individual terms then allows the $\alpha$ - and $\beta$-coefficients together with their associated time delays to be determined by inspection, thereby fully describing topology and circuit parameters of the recursive filter section.

The finite-element approximation applied to relationship (12) causes the original $j \omega$-axis to be mapped onto itself repeatedly. This can lead to disturbing aliasing effects. Application of this method thus tends to be confined to bandlimited situations for which the condition

$$
\left|\bar{H}_{R}(j \omega)\right| \approx 0 \quad \text { for }|\omega|>\omega_{s} / 2
$$

is satisfied.

2) Bilinear Transformation Method: The approach adopted here is to derive the recursive filter response from an appropriate lumped-element prototype response. This permits the wealth of information on prototype filters to be directly and conveniently exploited for present purposes. A bilinear-type transformation is thereby defined which establishes correspondence between the frequency variable $j \Omega$ in the lumped-element domain and the frequency variable $j \omega$ in the distributed domain, according to some chosen substitution formula $j \Omega=F(j \omega)$. Two examples for $F(j \omega)$ of particular interest are

$$
F(j \omega)=\frac{\Omega_{c}}{\tan \pi \omega_{c} / \omega_{s}} \cdot \frac{1-e^{-j 2 \pi \omega / \omega_{s}}}{1+e^{-j 2 \pi \omega / \omega_{s}}}
$$

and

$$
F(j \omega)=\Omega_{c} \cdot \tan \pi \omega_{c} / \omega_{s} \cdot \frac{1+e^{-\jmath 2 \pi \omega / \omega_{s}}}{1-e^{-\jmath 2 \pi \omega / \omega_{s}}} .
$$

These transformations are illustrated in Fig. 6. Unlike in the case of the impulse response method, the variable transformations result in a nonlinear distortion of the prototype frequency axis. In return, no aliasing effects occur.

To employ the prototype approach, the target response for the recursive transfer function $H_{R}(j \omega)$, as determined by the magnitude response of $G(j \omega) / H_{S}(j \omega)$, must first be translated into the $j \Omega$-domain. The magnitude of the resulting prototype target response is arrived at through application of the inverse frequency variable transforma-

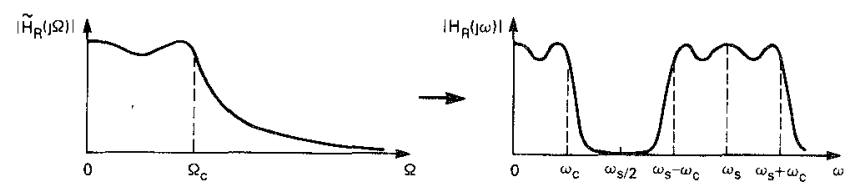

(a)

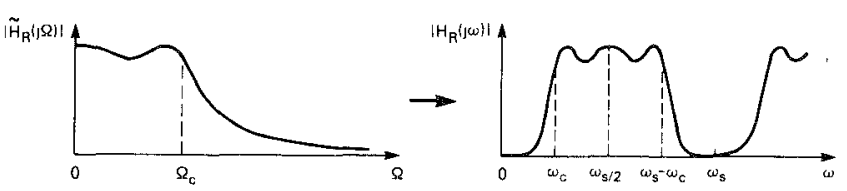

(b)

Fig. 6. Frequency variable transformations: (a) low-pass type, (b) highpass type.

tion to the corresponding $j \omega$-domain response by way of the substitution $j \omega=F^{-1}(j \Omega)$. Standard synthesis techniques are then utilized to find a rational function $\tilde{H}_{R}(j \Omega)$ in terms of the prototype-domain frequency variable that appropriately represents the prototype target response along the $j \Omega$-axis. Once such a function has been established, it is frequency-transformed back to the $j \omega$-domain to yield the recursive filter response

$$
H_{R}(j \omega)=\tilde{H}_{R}(F(j \omega))=\tilde{H}_{R}\left(e^{-j 2 \pi \omega / \omega_{s}}\right) .
$$

$H_{R}(j \omega)$ derived in this manner represents a rational function in $e^{-J 2 \pi \omega / \omega_{s}}$ that conforms with definition (9), with the values of the respective filter parameters again determined by inspection.

\section{B. Simplifying Approximations}

The $\alpha$ - and $\beta$-coefficients will, in general, represent a mix of positive and negative contributions, implying the need to reverse the phases of respective signal components over wide microwave frequency ranges. To avoid opposite sign terms, an approximation approach analogous to the one considered earlier for transversal filters may be employed, based on systematic application of substitution formula (7). The modified recursive structure that results must again be supplemented by a windowing filter in order to deal with out-of-band problems introduced by the approximation. The added windowing requirement can be accommodated quite simply, however, by appropriately tailoring the matching circuitries of individual active devices already assumed to be participants in the recursive process. Depending on the particular circumstances, this leads to composite filters that, overall, are less complex and are more easily implemented as microwave integrated circuits. The approximation process can be further extended to encompass deletion of the less significant terms. A numerical optimization of the resulting sparse structure is typically required to even out perturbations introduced by the approximations.

As follows from definition (9), a principal function of the feedforward terms in the numerator is to establish the zeros of transmission for the recursive filter. The implied reliance on transversal principles to achieve the zeros quite 


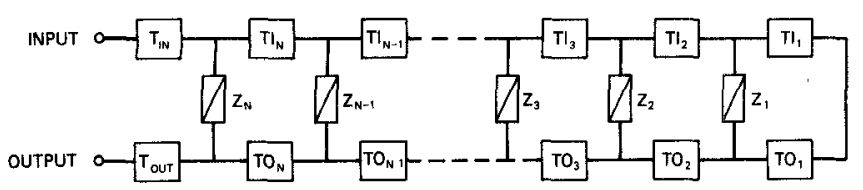

Fig. 7. Block diagram of a microwave recursive-type active filter.

often represents, however, an inefficient use of resources when compared to conventional passive filter alternatives. To implement such an alternative, the numerator of the recursive filter response (9) is split off and lumped in with the supplementary transfer function $H_{S}(j \omega)$ defined earlier. The design process is then adapted accordingly to arrive at a recursive solution that is made up of feedback terms exclusively.

\section{Physical Implementation}

A given recursive filter concept may be translated into an actual physical design in any number of ways. Possibilities are further increased through the option to combine recursive principles with conventional filter segments in pursuit of an optimally balanced overall design. Of particular interest in the present context are filter solutions that fit the block diagram format presented in Fig. 7. The respective circuits are composed of a chain of generalized two-port transmission elements which are augmented by feedback branches. The feedback elements labeled $Z_{n}$ merely consist of series-connected impedance elements, typically in the form of lumped resistors. As for the transmission elements, it is topologically convenient to distinguish between input and output components. They have been named $T I_{n}$ and $T O_{n}$, respectively. Optional elements $T_{\mathrm{IN}}$ and $T_{\text {OUT }}$ in cascade with the external ports have been included for generality.

The designated purpose of the regular transmission elements is to provide, in conjunction with the impedance elements $Z_{n}$, the necessary time delays for the various feedback loops in accordance with the individual exponential terms in the denominator of the rational function $H_{R}(j \omega)$, as well as to implement the corresponding loop gain values as defined by the $\beta$-coefficients. In order to impose a sense of direction on these loops, and thereby allow the feedback scheme to operate as intended, the levels of individual signals fed back through the nondirectional impedance elements must exceed, by a comfortable margin, the levels of parasitic contributions fed forward through the same elements. This is accomplished through assignment of gain functions to selected transmission elements, which introduces an appropriate differential between output- and input-related signal levels. In the simplest case, only the lowest order feedback loop need contain an active transmission element to boost output signal levels. But, active devices may be employed throughout the structure to either change relative signal levels, to obtain signal phase reversal and isolation, or to provide windowing.
The basic structure in Fig. 7 does not explicitly indicate any feedforward branches, and thus resembles the type of degenerate recursive filter mentioned earlier for which the zeros of transmission are realized with conventional passive filter segments rather than with less effective transversal-type sections. The passive nulling sections can be simply cascaded with the main recursive portion of the filter as provided for in Fig. 7 through the external transmission elements $T_{\text {IN }}$ and $T_{\text {OUT }}$. The preferred approach, however, is to absorb the passive functions directly into the feedback loop structure, assuming such to be compatible with pertinent time delay constraints imposed by the recursive process. Similar efforts should also be aimed at integrating windowing amplifiers into the loop structure, where not already required for establishment of signal level differentials within the structure. The internal transmission elements are thus assigned both amplitude-shaping and time delay responsibilities, simultaneously. In this way, the time delays associated with passive filter sections and windowing amplifiers are allowed to contribute in a constructive fashion to the delays already called for in the implementation of the recursive process. This leads to attractively compact filter realizations.

In a typical situation, the design procedure commences by deriving a set of parameters for the recursive transfer function (9) in accordance with overall filter specifications. One of the two methods outlined earlier may be employed to achieve this, whereby the supplementary function $H_{S}(j \omega)$ introduced in relationship (10) is initially set equal to unity. The recursive transfer function thus obtained serves as a preliminary assessment of circuit-related re-. quirements. The next step is to split off the numerator of expression (9), which is nominally responsible for providing the zeros of transmission, and approximate its transfer characteristics with suitable passive filter sections of conventional design. At the same time, it will be necessary to decide on how the remaining feedback portion of the filter is to be realized. This includes approximations aimed at reducing circuit complexity, the determination of pertinent windowing requirements, and the realization of such requirements with the help of band-limited amplifiers. Both the passive nulling function and the active windowing function, after'having been chosen appropriately, may then be represented by a composite supplementary function $H_{S}(j \omega)$. This function is used in defining a new recursive structure that contains feedback branches only and thus fits the format of Fig. 7. A set of modified recursive filter coefficients are derived with the help of the same techniques employed in the preliminary assessment. The final task is to translate the solution into reality by optimally placing both passive and active components within the context of the general topology and by numerically adjusting the circuit parameters for best overall performance.

The goal implicit in the above-outlined recursive approach is to seek efficient utilization of available transistors and of their capabilities. As in the case of the transversal-type filters discussed earlier, transistor-related constraints-such as limited gain, parasitics, and cost- 


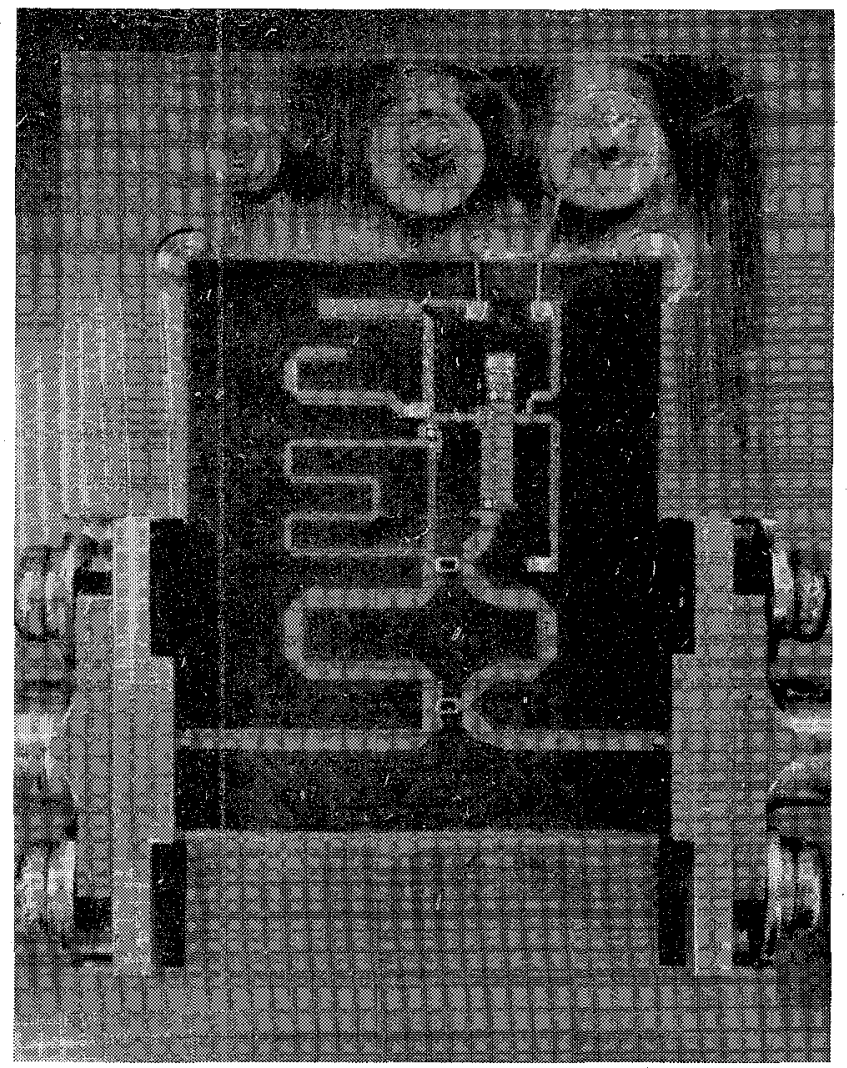

Fig. 8. Experimental $9-15-\mathrm{GHz}$ recursive-type bandpass filter, implemented on a $0.25-\mathrm{mm}$-thick fiberglass-reinforced Teflon substrate. The $50-\Omega$ input port is on the right and the $50-\Omega$ output port on the left.

become the critical factors impacting both analytical procedures and topological decisions. Compliance with these constraints invariably leads to designs which are individually tailored to specific application requirements. The design process itself is characterized by the wide range of issues that need be considered simultaneously. These include not only those related to the active devices, but also those dealing with the use of the simplifying approximations and passive filter supplements. The diversity and complexity of the issues leads to reliance on empirical judgements and computer-aided techniques, making it difficult to formulate a simple unified strategy. But, the following example should help illustrate the general idea.

\section{Experimental Example}

The various design aspects are illustrated in the following with an experimental filter example. For ease of comparison, the same overall objective was adopted as in the case of the transversal filter example, namely to achieve a 9-15-GHz triple-hump bandpass response, with stopbands spanning the frequency ranges from 5 to $8 \mathrm{GHz}$ and 16 to $19 \mathrm{GHz}$. The design was derived with the help of the high-pass-type bilinear transformation (16) which provides passband characteristics centered around $\omega_{s} / 4 \pi=12 \mathrm{GHz}$. Special effort was again focused on achieving the simplest possible filter solution consistent with the design objective. Besides adopting the degenerate recursive structure in Fig. 7, one-sign-only approximations based on substitutions (7)

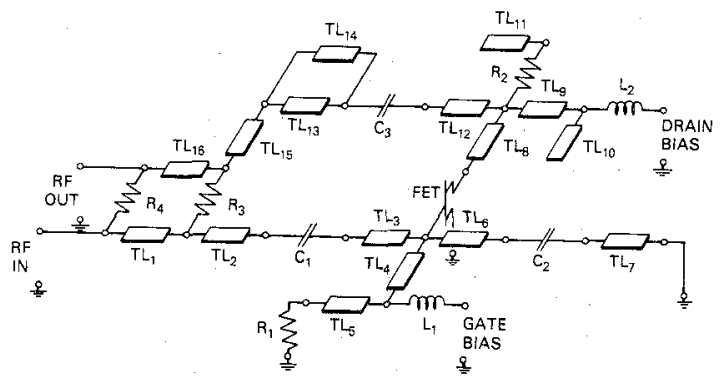

Fig. 9. Schematic circuit diagram of the experimental recursive-type bandpass filter. The values of characteristic impedance $Z_{0}$ and electrical length $\theta$ at $12 \mathrm{GHz}$ for the various uniform transmission-line segments are

$$
\begin{array}{llll}
T L_{1}: & Z_{0}=50 \Omega, \quad \theta=210^{\circ} & T L_{9}: & Z_{0}=75 \Omega, \theta=84^{\circ} \\
T L_{2}: & Z_{0}=50 \Omega, \theta=50^{\circ} & T L_{10}: & Z_{0}=50 \Omega, \theta=90^{\circ} \\
T L_{3}: & Z_{0}=35 \Omega, \quad \theta=78^{\circ} & T L_{11}: & Z_{0}=65 \Omega, \theta=180^{\circ} \\
T L_{4}: & Z_{0}=85 \Omega, \quad \theta=35^{\circ} & T L_{12}: & Z_{0}=50 \Omega, \theta=30^{\circ} \\
T L_{5}: & Z_{0}=85 \Omega, \quad \theta=100^{\circ} & T L_{13}: & Z_{0}=100 \Omega, \theta=90^{\circ} \\
T L_{6}: & Z_{0}=40 \Omega, \quad \theta=12^{\circ} & T L_{14}: & Z_{0}=100 \Omega, \theta=450^{\circ} \\
T L_{7}: & Z_{0}=40 \Omega, \quad \theta=32^{\circ} & T L_{15}: & Z_{0}=50 \Omega, \theta=10^{\circ} \\
T L_{8}: & Z_{0}=95 \Omega, \quad \theta=34^{\circ} & T L_{16}: & Z_{0}=50 \Omega, \theta=330^{\circ}
\end{array}
$$

The values of the lumped elements are

$$
\begin{array}{ll}
R_{1}=100 \Omega & C_{1}=C_{3}=10 \mathrm{pF} \\
R_{2}=25 \Omega & C_{2}=20 \mathrm{pF} \\
R_{3}=820 \Omega & L_{1}=L_{2}=15 \mathrm{nH} \\
R_{4}=680 \Omega &
\end{array}
$$

The transistor is a sub-half-micron Avantek M126 device

were again used, together with deletions of less significant terms. The recursive solution that resulted, comprising two feedback loops with affiliated feedback resistors, also required involvement of a windowing amplifier section and a passive filter segment to assist in defining the bandpass characteristics. At band center, the nominal effective electrical lengths of the loops, not including the frequencyindependent phase reversal introduced by the inverting amplifier, were $(5 / 2) \pi$ and $4 \pi$, respectively. The twobranch passive filter segment employed here is electrically identical to the one associated with the previous transversal example. Also, the gain section is virtually identical to the one described earlier, so as to facilitate comparative evaluation of the two types of circuits. In addition to accomplishing its windowing function, the amplifier is also needed to establish the required differential between input and output signal levels. Both the passive filter segment and the gain section are integral parts of all feedback loops. Their intrinsic time delays assume constructive roles in the recursive process, laying the foundation for a compact filter realization. The final design parameters for the composite circuit were obtained with the help of computer optimization to even out effects due to the approximations as well as circuit and active device parasitics.

The physical realization of the recursive filter is shown in Fig. 8. The circuit is implemented in microstrip form on a $0.25-\mathrm{mm}$-thick fiberglass-reinforced Teflon substrate, with coaxial $50-\Omega$ adapters at input and output. A corresponding schematic of the circuit is given in Fig. 9, together with 


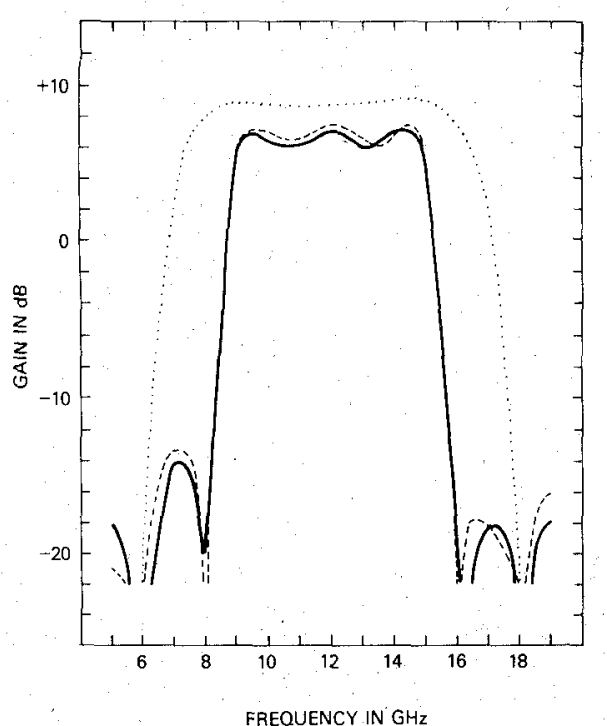

Fig. 10. Frequency characteristics of the recursive-type active filter example: —- measured filter response, -..- calculated filter response, ... calculated characteristics of the amplifier section by itself.

a list of individual circuit element values. The passive filter section is represented by transmission-line elements $T L_{13}$ and $T L_{14}$, and the gain section encompasses all circuit elements to the right of blocking capacitors $C_{1}$ and $C_{3}$. Transmission-line sections $T L_{1}, T L_{2}, T L_{15}$, and $T L_{16}$ provide the remainder of the required loop time delays, with resistors $R_{3}$ and $R_{4}$ serving as the two feedback resistors. Fig. 10 compares measured and calculated responses. Only a few minor transmission-line adjustments were involved in order to compensate for unpredictable variations in transistor characteristics. The circuit, once more, gets by with only one transistor, an Avantek M126 GaAs FET biased at half- $I_{D S S}$ and at a drain-source voltage $V_{D S}=$ $+3.0 \mathrm{~V}$. The transfer response of the amplifier section by itself is also shown. Its passband is intentionally designed to be wider than the actual filter response so as to reduce sensitivity of critical filter skirts to transistor-related tolerance effects.

Compared to purely transversal structures, the involvement of feedback in active recursive filters can complicate matters when it comes to evaluating noise and nonlinearity aspects. Due to the multiplicity of viable circuit configurations, it is quite difficult to formulate a general analysis that would carry much beyond some well-established and obvious guidelines on how to select transistor impedance matching conditions and bias levels. With the emphasis in the current study on basic design concepts, noise and nonlinearity consideration were consequently not included as design criteria. However, the current filter example, which relies on a single active element, presents a situation which lends itself to analysis with reasonable ease. Due to the nature of the recursive process, positive feedback alternates with negative feedback throughout the passband region as reflected by corresponding ripple swings in the frequency response of the filter. A reliable impression of how such a circuit behaves can be obtained through evaluation of its noise and nonlinear properties relative to the extreme points of feedback involvement.

As in the transversal example discussed earlier, the nonlinear behavior of the recursive filter example is mainly determined by the bias conditions for the transistor and its broad-band resistive output match. Although the presence of feedback introduces perturbational effects, these were not judged to be unique enough to justify the effort of pursuing nonlinear analysis and associated large-signal measurements. Noise figures were measured at one- $\mathrm{GHz}$ interyals within the $9-15-\mathrm{GHz}$ passband. The values decrease from a maximum reading of $7.8 \mathrm{~dB}$ at the lower band edge to a minimum of $5.2 \mathrm{~dB}$ at $14 \mathrm{GHz}$ before rising to $5.9 \mathrm{~dB}$ at the upper band limit. These numbers indicate a noise advantage for the recursive filter when compared to the previous figures for the transversal example. Part of this advantage could, of course, be regained by the latter through substitution of pre-amplification for post-amplification. Although not conclusive, the comparison is nevertheless interesting in that both examples use virtually identical gain and supplementary passive filter sections, with transistor chips even derived from the same wafer.

\section{Conclusions}

The main purpose of this study was to explore possibilities for effective utilization of microwave transistors in broad-band filter applications. Potential attractions offered by active filter approaches include the abilities to compensate for parasitic loss effects, to provide overall amplification, to reduce size, and to offer improved design flexibility. From a network synthesis point of view, one of the principal constraints on achieving broad-band performance is introduced by intrinsic time delays associated with transistors at microwave frequencies. This represents a severe impediment when attempting to adapt low-frequency active filter principles for use in the microwave range. For this reason, interest has been focused on transversal and recursive concepts which tend to be less constrained by time delay considerations.

Design approaches have been outlined for both transversal-type and recursive-type circuits. The overriding objective has been to derive filter concepts that can be readily translated into microwave integrated-circuit designs. Various simplifying approximations are used to achieve this, thereby leading to sparse structures that minimize the number of active devices required. Design effectiveness is further enhanced by including, where appropriate, conventional passive filter segments and windowing functions to assist the transversal and recursive processes. Experimental filter examples, comprising one of each kind, help to illustrate the concepts involved. Both examples share common performance goals in order to facilitate the comparison. They furthermore share designs for a passive filter segment and an amplifier section, which are utilized in similar ways in each of the examples. Despite these common factors, the two experimental examples possess distinct differences. As far as the achievement of a given frequency response with minimum effort is concerned, the 
recursive approach enjoys a significant advantage through availability of powerful feedback options. The recursive approach also tends to be more receptive to efficient integration of active devices and supplementary passive filter segments into the filter structure. This permits associated time delays to be used constructively, while allowing circuit dimensions to be minimized. Moreover, the type of recursive filter considered here does not require space-consuming junction elements as does its transversal counterpart. It is thus not surprising that the recursive approach evolves as the more attractive of the two solutions considered.

\section{ACKNOWLEDGMENT}

The author wishes to thank $H$. E. Heddings for his valuable assistance in assembling the test circuits, and $J$. R. Stiles of Avantek, Inc., for providing the transistor chips employed therein.

\section{REFERENCES}

[1] K. D. Adams and R. Y. Ho, "Active filters for UHF and microwave frequencies," IEEE Trans. Microwave Theory Tech., vol. MTT-17, pp. 662-670, Sept. 1969.

[2] R. V. Snyder and D. L. Bozarth, "Analysis and design of a microwave transistor active filter," IEEE Trans. Microwave Theory Tech., vol. MTT-18, pp. 2-9, Jan. 1970.

[3] H. Matsumura and Y. Konishi, "An active microwave filter with dielectric resonator," in 1979 IEEE MTT-S Int. Microwave Symp. Dig., pp. 323-325, April 1979.

[4] W. Jutzi, "Microwave bandwidth active transversal filter concept with MESFETs'," IEEE Trans. Microwave Theory Tech., vol. MTT19, pp. 760-767, Sept. 1971
[5] H. A. Willing, C. Rauscher, and P. deSantis, "A technique for predicting large-signal performance of a GaAs MESFET," IEEE Trans. Microwave Theory Tech., vol. MTT-26, pp. 1017-1023, Dec. 1978.

[6] C. Rauscher and H. A. Willing, "Simulation of nonlinear microwave FET performance using a quasi-static model," IEEE Trans. Microwave Theory Tech., vol. MTT-27, pp. 834-840, Oct. 1979.

[7] H. Y-F. Lam, Analog and Digital Filters. Englewood Cliffs, NJ: Prentice-Hall, 1979.

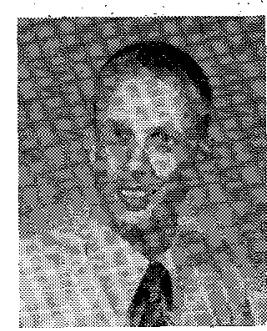

Christen Rauscher (S'73-M'75-SM'81) was born in Boston, MA, on November 4, 1944. He received the diploma in electrical engineering and the $\mathrm{Ph} . \mathrm{D}$ degree in 1969 and 1975 , respectively, both from the Swiss Federal Institute of Technology, Zurich, Switzerland.

From 1969 to 1976, he was employed as an Assistant and Research Associate at the Microwave Laboratory of the Swiss Federal Institute of Technology, where he conducted research on computer-aided tolerance optimization of microwave active circuits and on IMPATT power amplifiers. He held an international fellowship from the Swiss National Science Foundation from 1976 to 1978 . He spent this time at Cornell University, Ithaca, NY, and the Naval Research Laboratory, Washington, DC, investigating the nonlinear behavior of GaAs MESFET's. Since 1978, he has been employed at the Naval Research Laboratory, Washington, DC and is currently on sabbatical leave at the Los Alamos National Laboratory, Los Alamos; NM. His research interests are focused on nonlinear phenomena and optical signal interactions in microwave and millimeter-wave active semiconductor devices. 